Bartsch, Silke; Brandl, Werner

\title{
Von der Didaktischen Rekonstruktion zu einer Didaktik subjektorientierten Lernens und Lehrens
}

Haushalt in Bildung \& Forschung 4 (2015) 2, S. 116-125

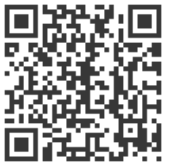

Quellenangabe/ Reference:

Bartsch, Silke; Brandl, Werner: Von der Didaktischen Rekonstruktion zu einer Didaktik

subjektorientierten Lernens und Lehrens - In: Haushalt in Bildung \& Forschung 4 (2015) 2, S. 116-125 URN: urn:nbn:de:0111-pedocs-203769 - DOI: 10.25656/01:20376

https://nbn-resolving.org/urn:nbn:de:0111-pedocs-203769

https://doi.org/10.25656/01:20376

in Kooperation mit / in cooperation with:

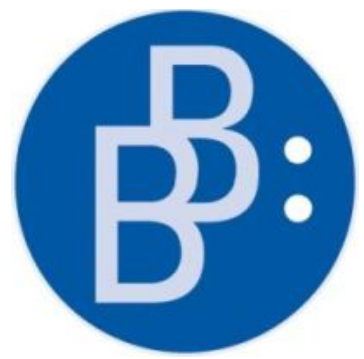

https://www.budrich.de

\section{Nutzungsbedingungen}

Gewährt wird ein nicht exklusives, nicht übertragbares, persönliches und beschränktes Recht auf Nutzung dieses Dokuments. Dieses Dokument is ausschließlich für den persönlichen, nicht-kommerziellen Gebrauch bestimmt. Die Nutzung stellt keine Übertragung des Eigentumsrechts an diesem Dokument dar und gilt vorbehaltlich der folgenden Einschränkungen: Auf sämtlichen Kopien dieses Dokuments müssen alle Urheberrechtshinweise und sonstigen Hinweise auf gesetzlichen Schutz beibehalten werden. Sie dürfen dieses Dokument nicht in irgendeiner Weise abändern, noch dürfen Sie dieses Dokument für öffentliche oder kommerzielle Zwecke vervielfältigen, öffentlich ausstellen, aufführen, vertreiben oder anderweitig nutzen.

Mit der Verwendung dieses Dokuments erkennen Sie die Nutzungsbedingungen an

\section{Terms of use}

We grant a non-exclusive, non-transferable, individual and limited right to using this document.

This document is solely intended for your personal, non-commercial use. Use of this document does not include any transfer of property rights and it is conditional to the following limitations: All of the copies of this documents must retain all copyright information and other information regarding legal protection. You are not allowed to alter this document in any way, to copy it for public or commercial purposes, to exhibit the document in public, to perform, distribute or otherwise use the document in public.

By using this particular document, you accept the above-stated conditions of use.

\section{Kontakt / Contact:}

\section{peDOCS}

DIPF | Leibniz-Institut für Bildungsforschung und Bildungsinformation Informationszentrum (IZ) Bildung

E-Mail:pedocs@dipf.de

Internet: www.pedocs.de

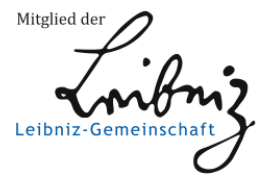


4. Jahrgang Heft 2 2015

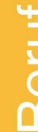

m

(1)

$\frac{\sqrt{2}}{\frac{2}{c}}$

$>$

(1)

(1)

ते

(1)

है

(1)

$\frac{1}{2}$

3

$>$

(1)

$\frac{8}{8}$

$\frac{1}{c}$

है

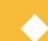

필

$\checkmark$

$\frac{\overline{1}}{\frac{1}{0}}$

है

(1)

(1)
है

$\frac{d}{\frac{c}{c}}$

\section{Haushalt in}

Forschung

Wa(h)re Gesundheit
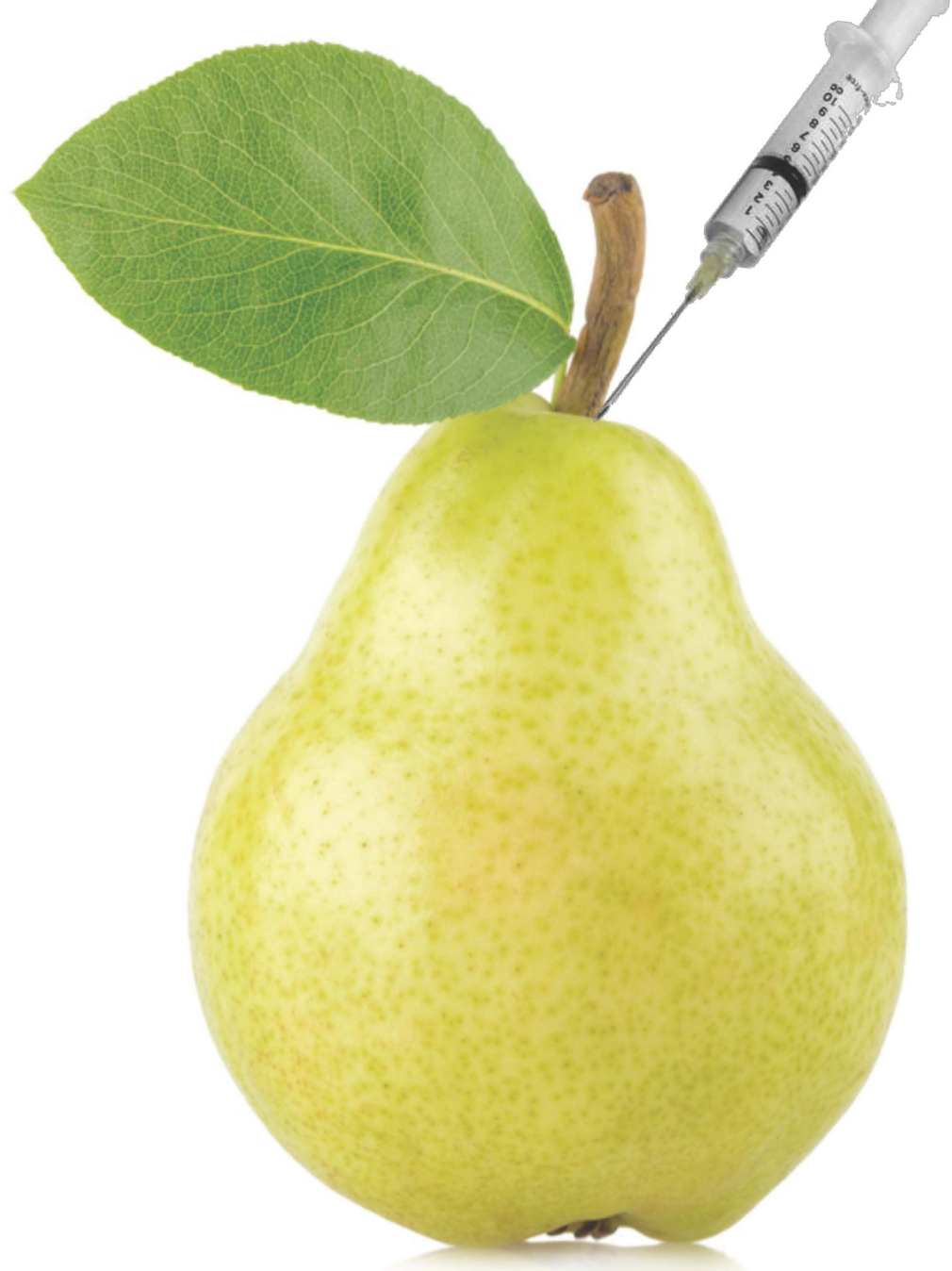
Inhaltsverzeichnis |

Gabriela Leitner

Editorial...... 2

Gabriela Leitner \& Maria Schuh

Ernährungs- und Verbraucherbildung - quo vadis? 3

Kirsten Schlegel-Matthies

Gesundheit und Selbstverantwortung - Was kann und was sollte gelehrt werden?. 18

Angela Häußler

Lässt sich Gesundheit lehren?

Ute Bender

„Eating Smart“ - Funktionen von Ernährung in der (schulischen)

Gemeinschaftsverpflegung.

Ursula Buchner

Lernwege in der Schulküche 58

Workshopnachlese

Theres Rathmanner

Ernährungsprojekte in Schulen weltweit - ein Blick über den Tellerrand 86

Michaela Kropatschek

Spielregeln für Werbung im Lebensmittelbereich und in der Schule.

Maya Lucia Joray, Michèle Simone Leuenberger \& Zeno Stanga

Ursachen und Strategien zur Bekämpfung von Übergewicht und Adipositas

bei Kindern und Jugendlichen - aus der Sicht der Schule.

Silke Bartsch \& Werner Brandl

Von der Didaktischen Rekonstruktion zu einer

Didaktik subjektorientierten Lernens und Lehrens.

Rezensionen

Thomas Mohrs

Nachhaltige Lebensstile. 126

Regine Bigga

Was der Mensch essen darf. 


\section{Von der Didaktischen Rekonstruktion zu einer Didaktik subjektorientierten Lernens und Lehrens}

Das „Modell der Didaktischen Rekonstruktion“ stellt einen praxistauglichen Entwicklungsund Forschungsrahmen für naturwissenschaftlichen Unterricht dar. Es stellt sich die Frage, ob und - wenn ja - wie das Modell für lebensweltbezogene Inhalte der Ernährungs- und Verbraucherbildung (EVB) genutzt werden kann. Für die Konzeption einer subjektorientierten Didaktik in der EVB wird hier ein adaptiertes Modell skizziert und zur Diskussion gestellt.

Schlüsselwörter: Subjektorientierung, Didaktische Rekonstruktion, Kompetenz, Konzept, Kontext

\section{Einleitung: Didaktik subjektorientierten Lernens und Lehrens in der EVB}

Unterricht in lebensweltbezogenen Fächern stellt schnell einen Spagat zwischen im Alltag bewährten „Laientheorien“ und „Wissenschaftstheorien“" dar, häufig mit dem zweifelhaften „Erfolg“, dass die Lernenden Alltag und Schule gedanklich trennen. Auf der Suche nach tragfähigen fachdidaktischen Konzeptionen rücken u. a. Präkonzepte (und subjektive Theorien) von Lernenden, aber auch die von Lehrenden in den Fokus der Überlegungen und fachdidaktischer Diskussionen ${ }^{2}$ (vgl. Bartsch \& Methfessel, 2014; Brandl, 2014; Wespi \& Senn Keller, 2014).

Das Modell der „Didaktischen Rekonstruktion“ (Kattmann et al., 1997; Gropengießer \& Kattmann, 2013) ist ein etablierter Entwicklungs- und Forschungsrahmen für fachdidaktische Frage- und Problemstellungen ${ }^{3}$ insbesondere in den Naturwissenschaften. Das Modell bezieht Präkonzepte von Schülerinnen und Schülern systematisch in die Planung von Unterricht ein. Entsprechend stellt sich die Frage: Was kann das Modell der „Didaktische Rekonstruktion“ nach Kattmann et al. (1997) für die EVB leisten? Wie müsste es für die EVB spezifiziert werden?

Neben der Klärung des Subjektbegriffs und dessen didaktiktheoretischer Verortung müssen dazu auch die grundlegenden Begriffe und zugrundeliegenden Konzepte transparent gemacht werden. Dazu wurde auf dem Workshop in Linz ${ }^{4}$ gearbeitet. Um zu einer Konzeption einer subjektorientierten Didaktik zu kommen, wurde daran anschließend ein adaptiertes Modell entwickelt und skizziert, das hier zur Diskussion gestellt wird. 
Subjektorientierte Didaktik |

\section{Didaktische Rekonstruktion: Intentionen, Chancen und Restriktionen}

Um diese Fragen zu beantworten, wird zunächst das Modell der „Didaktischen Rekonstruktion“ (Kattmann et al., 1997; Gropengießer \& Kattmann, 2013) in aller Kürze in seiner Bedeutung für den naturwissenschaftlichen Unterricht vorgestellt, um daran anschließend Möglichkeiten und Grenzen des Modells für die EVB auszuloten.

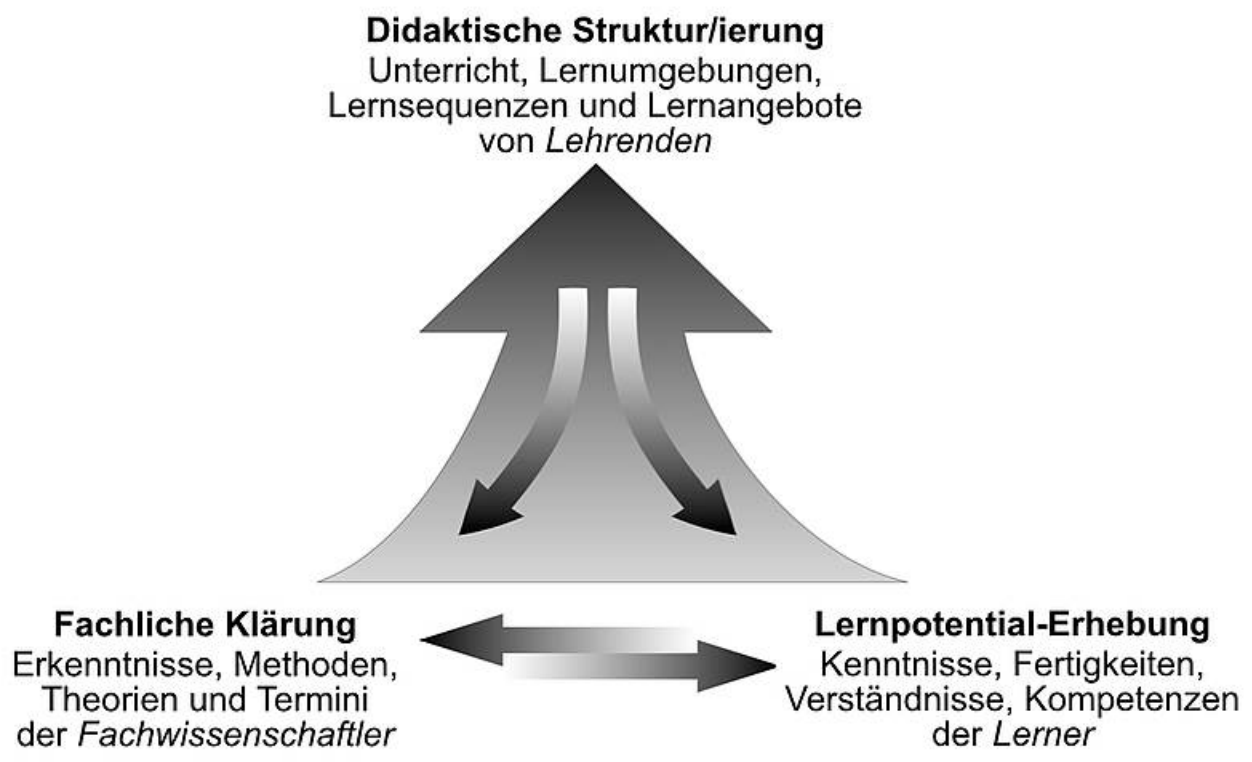

Abb. 1: Das Modell der Didaktischen Rekonstruktion (Quelle: mit freundlicher Genehmigung aus Gropengießer \& Kattmann, 2013)

„Ziel dieses Modells ist es, auf der Basis von Schülervorstellungen und fachlicher Klärung Lernumgebungen zu konstruieren“" (van Dijk \& Kattmann, 2010, S. 8). Im schulischen Unterricht geht es darum, Unterrichtsinhalte nicht unmittelbar und unverändert dem Wissenschaftsbereich zu entnehmen, sondern sie müssen vielmehr mit pädagogischer Zielsetzung erst hergestellt, $\mathrm{d}$. $\mathrm{h}$. didaktisch rekonstruiert werden. Dies ist nach Kattmann et al. (1997, S. 4) mehr als:

- ein effektives, methodisches Umsetzen oder motivierendes Einkleiden von wissenschaftlicher Erkenntnis,

- eine didaktische Reduktion, die vorwiegend den Prozess bezeichnet, mit dem aus der im Wissenschaftsbereich vorhandenen Fülle ausgewählt wird, um einen angemessenen Umfang herzustellen oder/und um (häufig falsch) zu vereinfachen, 


\section{Subjektorientierte Didaktik}

- eine didaktische Transformation, die den Prozess betrifft, mit dem die Form der Darstellung und das Niveau der experimentellen Fertigkeiten den Schülerinnen und Schülern angepasst werden, so dass der Unterrichtsgegenstand verständlich und für sie fassbar wird.

Damit wird dem Umstand Rechnung getragen, dass die Lernenden nicht über mehr oder weniger „defizitäre“ Vorstellungen über diese Gegenstandsbereiche - sog. Präkonzepte - verfügen ${ }^{5}$, die es im Rahmen geeigneter Lernumgebungen gilt, den wissenschaftlichen Konzepten anzunähern bzw. durch sie zu ersetzen. Eine solch radikaler Vorstellungswandel von „falschen“ Prä- zu „richtigen“ Konzepten, einem Konzeptwechsel (conceptual change; vgl. Strike \& Posner, 1992; Duit \& Treagust, 2003) basiert ,,auf einem Verständnis lebensweltlicher Vorstellungen als Fehlvorstellungen und ist deshalb nicht ohne weiteres mit einem konstruktivistischen Verständnis von Lernen zu vereinbaren“ (Schwanewedel, 2011, S. 18) bzw. ist in der Folge ein „didaktischer Exorzismus, bei dem es um auslöschen, bekämpfen, abbauen oder vergessen geht" (Gropengießer, 2006, S. 96).

Schülervorstellungen sind demnach nicht einfach als unwissenschaftlich ,fehlerhaft" und wissenschaftliche Vorstellungen als fehlerfrei „richtig“ anzusehen, vielmehr sind sie aus ihrem jeweiligen Kontext, den situativen Bedingungen und in ihrer besonderen Funktion einzuordnen.

Tab. 1: Vorstellungen zur Genetik (Quelle: Eigene Darstellung mit Angaben aus Schwanewedel, 2011)

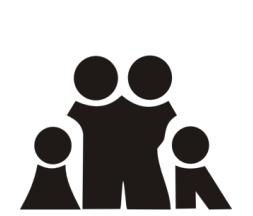

Lebensweltliche Vorstellungen

- Vererbung von Merkmalen: Nase, Gesicht, Größe etc.

- Verteilung von elterlichen Eigenschaften*

- Gene als autonome Lebewesen

- Gene als Behälter

- Gene als Krankheitsträger

- $\quad .$.

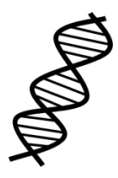

Wissenschaftliche Konzepte
- Vererbung: Weitergabe von Genen

- Genotyp: die in den Genen festgelegten Erbinformationen

- Phänotyp: das äußere Erscheinungsbild eines Organismus

- Gene als Konstrukte

•

* frei nach J. W. v. Goethe: Vom Vater hab ich die Statur, vom Mütterchen die Frohnatur!

Dass der wissenschaftliche Fortschritt in den Naturwissenschaften auch auf einer Abfolge differenter und divergierender Vorstellungen über denselben Gegenstand 


\section{Subjektorientierte Didaktik |}

fußt, lässt sich am Beispiel der Genetik sehr deutlich darstellen: Die mit den Mendel'schen Kreuzungsversuchen auf der phänomenologischen Ebene aufgezeigte Regelhaftigkeit von Vererbung ist zwischenzeitlich auf der molekularen Ebene als Genregulation angelangt - und selbst damit dürfte der „Wandel im Verständnis von Genen" (Schwanewedel, 2011, S. 308) noch nicht beendet sein, wie die neueren Erkenntnisse der Epigenetik nahelegen, die ein lang gehegtes Dogma in Frage stellen, dass nämlich die Eigenschaften eines Organismus durch das vererbte Genmaterial unveränderbar bestimmt seien.

Das Modell der „Didaktischen Rekonstruktion“ entfaltet seine Wirksamkeit insbesondere in naturwissenschaftlichen Fächern, deren Gegenstandsbereich sich in klar begrenzten und beschreibbaren fachwissenschaftlichen Konzepten darstellen lässt. Mit den Projekten Naturwissenschaften im Kontext in Gestalt von piko (Physik im Kontext), $\mathrm{CHiK}$ (Chemie im Kontext) und BiK (Biologie im Kontext) ist eine fächerübergreifende Grundlegung einer Allgemeinen Fachdidaktik für diese Naturwissenschaften erfolgt. Ermöglicht wird dies zum einen durch eine gemeinsam geteilte Fokussierung auf sogenannte Basiskonzepte des je spezifischen Fachwissens und zum anderen die ebenfalls geteilte Problematik, das in einer Fachsystematik vorliegende Wissen an die lebensweltlichen Kontexte anbinden zu müssen: Sie verfolgen damit das Ziel, naturwissenschaftliche Konzepte und Arbeitsweisen in der unterrichtlichen Umsetzung an das Vorwissen und die Alltagserfahrungen sowie die wissenschaftlichen Anwendungsfelder anzubinden, sodass damit die Kompetenzentwicklung der Schülerinnen und Schüler gefördert werden kann.

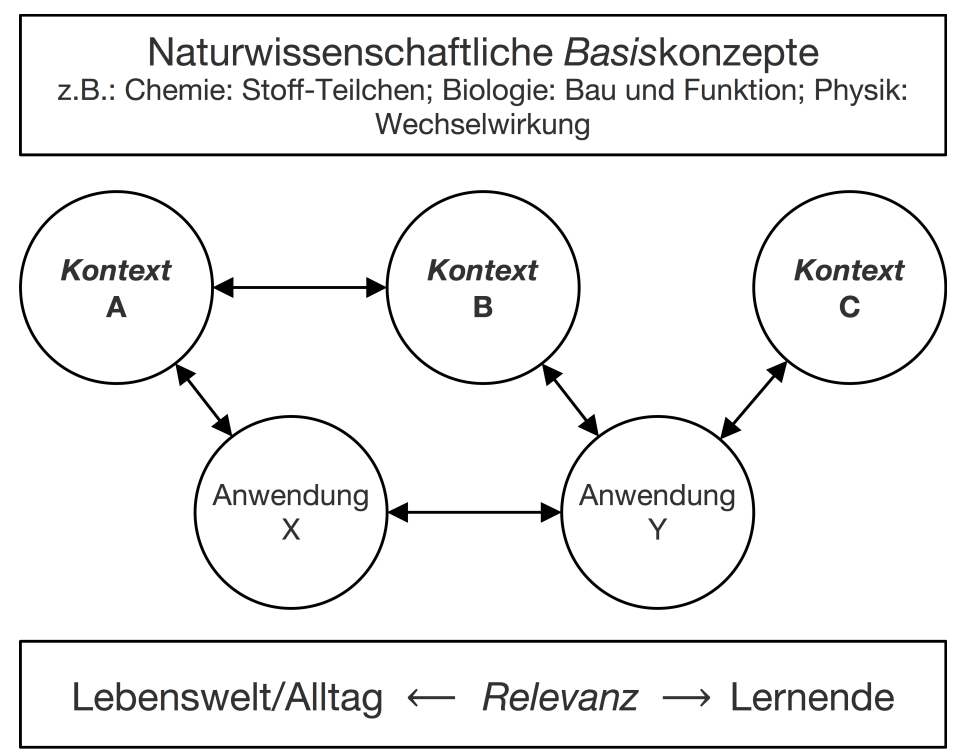

Abb. 2: Naturwissenschaftliche Konzepte \& Kontexte (Quelle: Eigene Abbildung in Anlehnung an Muckenfuß, 2004, S. 65) 


\section{Subjektorientierte Didaktik}

Auf der Basis ähnlich strukturierter Basiskonzepte und der notwendigen Kontextualisierung von Phänomenen der Lebenswelt und subjektiven Relevanz für die Lernenden, erweist sich eine solchermaßen auf die naturwissenschaftliche Domäne beschränkte Allgemeine Fachdidaktik als tragfähig.

Mit Kontexten sind Themen - oder thematische Aspektierungen eines fachlichen Teilgebietes - gemeint, mit denen jeweils ein wohl definierter Bereich der wissenschaftlichen Ideenwelt erschließbar ist. Diese Kontexte greifen - wie andere Themen auch - Inhalte oder Probleme aus der Lebenswelt auf, die für die Adressaten unseres Unterrichts subjektiv möglichst bedeutungsvoll sind. (Muckenfuß, 2004, S. 64).

Für die Ernährungs- und Verbraucherbildung zeigen sich dabei zwei Herausforderungen: Die Inter- und Transdisziplinarität der EVB und deren Alltagsnähe. In der Ernährungs- und Verbraucherbildung mit ihrer Vielfalt an Bezugswissenschaften (um nur ein paar - und oftmals sogar nur im Plural - zu erwähnen: Haushaltswissenschaften, Ernährungswissenschaften, Ernährungspsychologie, Soziologie, Biologie, Ernährungsmedizin etc.) ist die eine Allgemeine Fachdidaktik, die allen Facetten einer Ernährungs-, (Gesundheits-) und Verbraucherbildung gerecht werden kann, denkbar unwahrscheinlich zu konzeptionieren. Zwar ist die EVB in ähnlicher Weise mit dem Problem der Fachsystematik und des lebensweltlichen Kontextes konfrontiert, allerdings in etwas veränderter Form:

1. Während die Naturwissenschaften den lebensweltlichen Kontext quasi erst suchen müssen, ist dieser in der EVB ,verstrickt“.

2. Damit stellt sich nicht nur die Frage nach dem „Vorwissen“ der Lernenden, sondern auch die nach den Präkonzepten und subjektiven Theorien der Lehrenden.

3. Gleichzeitig sind „defizitäre“ Konzepte nicht so einfach auszumachen, da Widersprüche zum Alltag gehören und häufig nicht auflösbar sind.

Beispielsweise können individuell tragfähige Konzepte (z. B. nach einer bedarfsgerechten Ernährung) mit gesellschaftlich erwünschten Konzepten (z. B. nachhaltige Ernährungsweisen zur Herstellung einer Ernährungssicherheit für alle Menschen) kollidieren. Ziel kann daher nicht die Ausblendung von Widersprüchen sein, aber auch daraus resultiert die Frage: „Welche Präkonzepte sind erlaubt?“. Hier geht es um die Reflektion der normativen Setzungen des Faches (vgl. dazu Brandl, 2014). Werden keine Normen gesetzt, besteht die Gefahr der Beliebigkeit, die wiederum in eine Sackgasse führt.

\section{3 ... Adaption und Optionen für die EVB}

Anders als in den Naturwissenschaften, bei denen eine „Konzeptänderung“ durchaus. zielführend sein kann, gibt es in der Ernährungs- und Verbraucherbildung meist 


\section{Subjektorientierte Didaktik |}

mehrere gleichberechtigte Konzepte, die unterschiedliche Bedeutungen und/oder Funktionen haben bzw. im Alltag widersprüchlich sein können. Daher ist zunächst eine Klärung der Ebenen im Lernprozess notwendig: Geht es um das Verständnis eines Konzeptes, dann kann das originäre „Modell der Didaktischen Rekonstruktion“ hilfreich sein. Oder geht es um Konzepte, die in Alltagssituationen für Handlungen verfügbar sind. Hier stößt das Modell an Grenzen, weil fachwissenschaftliche Perspektiven im Alltag i. d. R. nicht widerspruchsfrei sind. So finden Alltagshandeln und Entscheidungen nicht unter Ausblendung anderer (z. T. widersprüchlicher) Konzepte statt und auch einzelne Konzepte sind komplex. Beispiel: Selbstbacken von Brot mit frischgemahlenem, Korn aus regionalem Anbau bereits unter der Perspektive der Nachhaltigkeit erzeugt unterschiedliche Bewertungen. Das führt i. d. R. dazu, dass in der EVB Handlungs- und Entscheidungskompetenzen gefördert werden, die begründet, aber nicht widerspruchsfrei sein können.

Didaktische Strukturierung

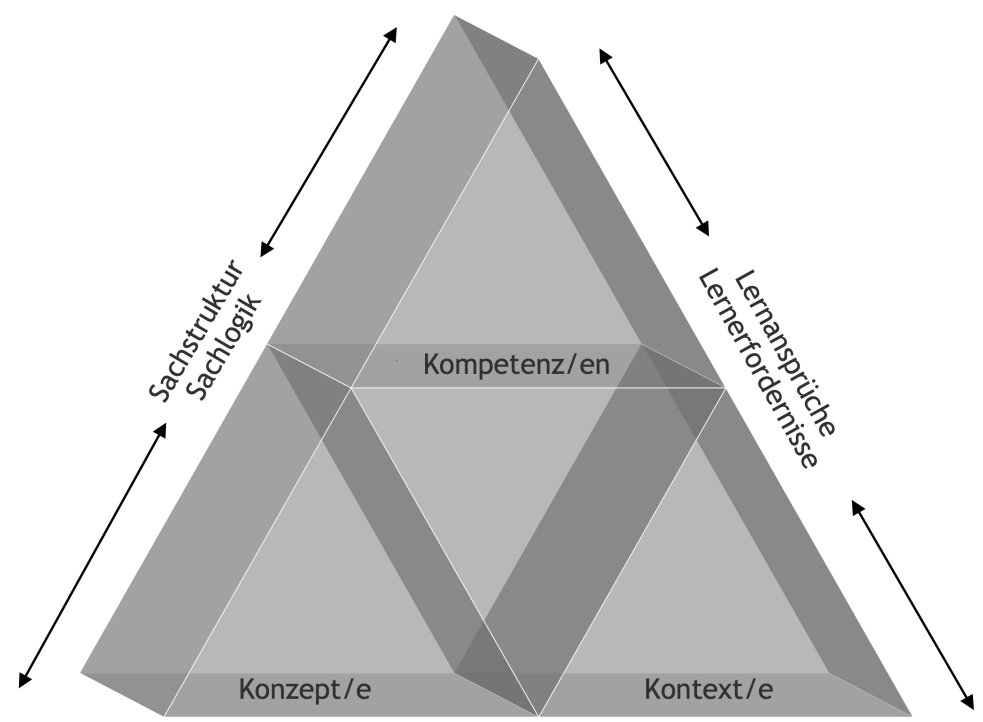

Fachsystematik

Subjektorientierung

lebensweltliche Vorstellungen

Abb. 3: Subjektorientierung - didaktisch fragil-stabil (Quelle: Eigene Darstellung)

Die im Modell der „Didaktischen Rekonstruktion“ als wesentliche fachdidaktische Problemstellung konzipierte

- Erfassung der Lernerperspektiven, d. h. insbesondere das alltägliche oder lebensweltliche Wissen der Lernenden, deren Vorstellungen, Interessen etc. sowie die 


\section{Subjektorientierte Didaktik}

- fachliche Klärung, d. h. die kritische Untersuchung der Fachsystematik/en, des fachlichen Wissens, der wissenschaftlichen Positionen und der zugrundeliegenden Theorien unter dem Aspekt der Vermittlung und die

- didaktische Strukturierung, d. h. die Entscheidung auf Basis der erfassten Lernerperspektiven und der fachlichen Klärung, mit welchem Ziel, welches Thema, in welchem Umfang, mit welchem Schwierigkeitsgrad, in welchen Bezügen Unterricht gestaltet werden kann/muss,

bleiben als zentrale fachwissenschaftliche, fachdidaktische und fachmethodische Aufgabenstellungen auch und gerade in der Ernährungs- und Verbraucherbildung bestehen. Der „subjektive Faktor" nimmt in einem lebensweltorientierten Fach/Fächerkanon und der Konzeption einer Didaktik subjektorientierten Lernens und Lehrens darüber hinaus eine prominente Rolle ein (vgl. Bartsch \& Methfessel, 2014; Brandl, 2014). Die lebensweltlichen Vorstellungen strukturieren nicht nur auf einer theoretischen Ebene Einstellungen zu Alltagshandeln und Lebensgestaltung, sondern nehmen unmittelbar Einfluss auf die praktische Ausgestaltung - mit allen positiven, wie auch möglicherweise negativen Konsequenzen, ob es nun um (,gesunde") Ernährung, (nachhaltigen) Konsum, (bezahlbares) Schuldenmachen oder auch (klimaschonende) Mobilität geht. Außerdem verlangen die daraus resultierenden Lernansprüche bzw. Lernerfordernisse, dass die Fachsystematik in ihrer Sachstruktur und Sachlogik mit der Psychologik der Lernenden in der didaktischen Strukturierung des Lerninhaltes und der Lernumgebung kompatibel ist bzw. gemacht werden kann.

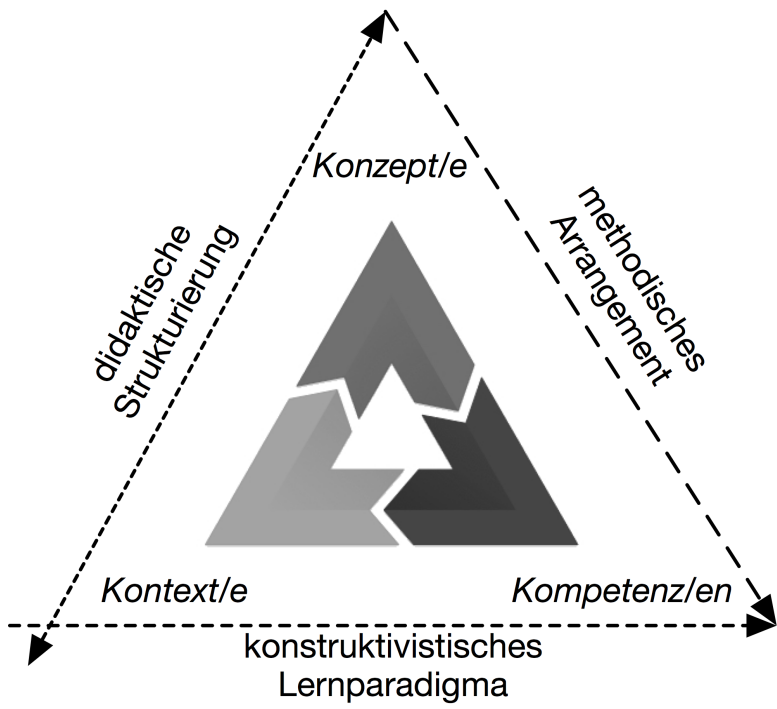

Abb. 4: Konstruktivismus - Kontext - Konzept - Kompetenz (Quelle: Brandl, 2014, S. 43) 


\section{Subjektorientierte Didaktik}

Die paradigmatisch konstruktivistische Ausrichtung der „Didaktischen Rekonstruktion“ ist daher „im Kern“ dahingehend zu ergänzen bzw. zu präzisieren, dass die didaktische Strukturierung und das methodische Arrangement unter Einbezug der Konzepte und Kontexte auf den Kompetenzerwerb bzw. die Kompetenzentwicklung fokussiert wird.

\section{Ausblick: Perspektiven einer Didaktik subjektorientierten Lernens und Lehrens}

Für die Entwicklung der Perspektive einer Didaktik subjektorientierten Lernens und Lehrens in der Ernährungs- und Verbraucherbildung stellt sich daher zunächst die Frage nach verbindlichen Basiskonzepten, die auf der Basis des REVISRahmencurriculums zu diskutieren sind. Ein Basiskonzept stellt die „Nachhaltige Ernährung" dar. Es eignet sich für die Theoriebildung, weil es die angesprochenen domänentypischen Herausforderungen enthält. So gibt es mehrere tragfähige fachwissenschaftliche Konzepte, die zu Entscheidungsdilemmata führen können. Darüber hinaus trifft man auf Vorstellungen der Lehrenden (und Lernenden), die häufig normativ sind und/oder tragende Teilaspekte ausblenden und damit Alltagsentscheidungen (dogmatisch) vereinfachen und meist nicht im Sinne eines kompetenten, reflektierten Handelns getroffen werden. Hierzu müssen weitergehende hochschuldidaktische Forschungen getätigt und didaktisch-methodische Konzepte überdacht und weiterentwickelt werden. Das Modell der Didaktischen Rekonstruktion bietet hierzu einen anschlussfähigen Theorierahmen für die Erfassung des PCK („Pedagogical Content Knowledge“; Shulman, 1986) der Lehrenden.

\section{Anmerkungen}

1 Zur Differenzierung der Begriffe siehe Bartsch \& Methfessel, 2014

2 Heft 3/2014 der Haushalt in Bildung \& Forschung über die „Subjektorientierung in der Ernährungs- und Verbraucherbildung" eignet sich zur vertiefenden Lektüre.

3 Einen Überblick über die Spannbreite und Differenzierung des Einsatzes der Didaktischen Rekonstruktion als Forschungs- und Entwicklungsrahmen findet man in der Schriftenreihe Beiträge zur Didaktischen Rekonstruktion:

[www.uni-oldenburg.de/diz/publikationen/beitraege-zur-didaktischenrekonstruktion]

4 Die Diskussion im Rahmen des Workshops „Begriffe und Konzepte rund um die Subjektorientierung“ der 3. D-A-CH-Tagung am 13./14. Februar 2015 in Linz dazu war sehr fruchtbar. Wir danken den Teilnehmenden des Workshops für die wertvollen Anregungen.

5 Zur systematischen Differenzierung des Begriffes siehe Wilhelm (2012). 


\section{Subjektorientierte Didaktik}

6 Bartsch, S. (in Vorbereitung). Alltagsvorstellungen von Studierenden zu Nachhaltiger Ernährung (Arbeitstitel).

\section{Glossar}

didaktische Reduktion: Vereinfachung der komplexen Wirklichkeit auf elementare Sachverhalte, um sie für die Lernenden überschaubar und begreifbar zu machen didaktische Rekonstruktion: Orientierungsrahmen zur Planung, Durchführung und Auswertung von Lern-Lehrprozessen, in denen Alltagsvorstellungen und fachliches Wissen aufeinander bezogen werden

Kompetenz/en: sind erlern- und verfügbare kognitive Fähigkeiten und Fertigkeiten, einschließlich entsprechender motivationaler, volitionaler, sozialer Bereitschaften, in variablen Situationen Problemlösungen erfolgreich und verantwortungsvoll herbeizuführen

Kontext/e: in Bezug auf die Lebenswelt persönlich oder gesellschaftlich relevante Anknüpfungspunkte an Alltagserfahrungen und Vorwissen bzw. wissenschaftliche Anwendungsfelder

Konzept/e: mentale Konstrukte und Repräsentationen als Ordnungsschema für den systematischen und kumulativen Aufbau von Wissen und Verständnis

Konzeptänderung (conceptual change): Veränderung bestehender Vorstellungen, gefasst als Prozess, der von alltagstauglichen vorwissenschaftlichen Präkonzepten hin zu tragfähige(re)n wissenschaftlichen $\rightarrow$ Konzepten führt

\section{Literatur}

Bartsch, S. \& Methfessel, B. (2014). „Der subjektive Faktor“. Bildung in einem lebensweltorientierten Fach. Haushalt in Bildung \& Forschung, 3(3), 3-32.

Brandl, W. (2014). Bausteine und Baustelle einer Didaktik subjektorientierten Lernens und Lehrens. Haushalt in Bildung \& Forschung, 3(3), 33-53.

Duit, R. \& Treagust, D. (2003). Conceptual Change: A powerful framework for improving science teaching and learning. International Journal of Science Education, 25(6), 671-688. [DOI:10.1080/09500690305016].

Gropengießer, H. (2006). Wie man Vorstellungen der Lerner verstehen kann. Lebenswelten - Denkwelten - Sprechwelten (Beiträge zur Didaktischen Rekonstruktion 4, 2. Aufl.). Oldenburg: Didaktisches Zentrum.

Gropengießer, H. \& Kattmann, U. (2013). Didaktische Rekonstruktion. In H. Gropengießer, U. Harms \& U. Kattmann (Hrsg.), Fachdidaktik Biologie (9. Aufl., S. 16-23). Hallbergmoos: Aulis Verlag. 


\section{Subjektorientierte Didaktik |}

Kattmann, U. Reinders, D., Gropengießer, H. \& Komorek, M. (1997). Das Modell der Didaktischen Rekonstruktion - Ein Rahmen für naturwissenschaftsdidaktische Forschung und Entwicklung. ZFDN, 3(3) 3-18.

Muckenfuß, H. (2004). Themen und Kontexte als Strukturelemente des naturwissenschaftlichen Unterrichts. Zu den Schwierigkeiten, systematisches Physiklernen zu organisieren. Physik und Didaktik in Schule und Hochschule, 3(2), 57-66.

[http://phydid.physik.fu-berlin.de/index.php/phydid/article/view/23/23].

Schwanewedel, J. (2011). Biologie verstehen. Gene und Gesundheit (Beiträge zur Didaktischen Rekonstruktion 32). Oldenburg: Didaktisches Zentrum.

Shulman, L. S. (1986). Those who understand: Knowledge growth in teaching. Educational Researcher, 15(2), 4-31. [DOI: 10.3102/0013189X015002004].

Strike, K.A. \& Posner, G.J. (1992). A revisionist theory of conceptual change. In R.A. Duschl \& R.J. Hamilton (Eds.), Philosophy of science, cognitive psychology and educational theory and practice. (pp. 147-176). New York (NY): State University of New York Press.

Wilhelm, M. (2012). Kompetenzorientierten Unterricht konzipieren - am Beispiel der Naturwissenschaften. Haushalt in Bildung \& Forschung, 1(3), 15-30.

\section{Verfasserin/Verfasser}

Prof. ${ }^{\text {in }}$ Dr. Silke Bartsch

Pädagogische Hochschule Karlsruhe

Bismarckstr. 10

D-76133 Karlsruhe

E-Mail:

silke.bartsch@jugendesskultur.de

Internet:

www.jugendesskultur.de
Werner Brandl M.A.

Institutsrektor

Staatsinstitut für die Ausbildung von Fachlehrern

- Abteilung II -

Am Stadtpark 20

D-81243 München

E-Mail:wbrandl@stif2.de

Internet: www.stif2.de 